\title{
An Analysis of the Character Tris in Divergent in the Light of Faciality Wei Wei
}

\section{School of Foreign Languages, Wuhan University of Science and Technology, Wuhan 430063,} China

\begin{abstract}
Faciality is a postmodern philosophy jointly proposed by French postmodern philosophers Deuleze and Guattari. It can be reflected in Deleuze and Guattari's work-A Thousand Plateaus: Capitalism and Schizophrenia, which studies on Capitalism and Schizophreniahas and has deep influences on contemporary western scholars. "Face" is a system composed of "black hole" and "white wall", which is formed by the change of the socioeconomic structure and its power management. This essay will analyze the Character Tris in Veronica Rose's Divergent based on the theory of Faciality; and will further analyze how Tris from "face" realizing her physical and pychological changes through "rhizome", "deterritorialization", and "the body without organs".
\end{abstract}

Keywords: Divergent; Tris; Faciality; Rhizome; Deterritorialization; Body without organs

\section{小说《分歧者》中人物翠丝的“脸面性”解读}

\author{
魏巍 \\ 武汉科技大学外国语学院、英语系, 湖北省 武汉市 430063
}

摘要: 在法国后现代主义哲学家德勒兹的政治哲学体系中, “脸”是其核心概念之一, 它贯穿了德勒兹与伽塔里对当代西 方思想界深具影响力的一部研究资本主义与精神分裂症的著作———高原》, 并与“欲望机器”, “无器官身体”, “解辖域 化”等概念密切相关。它是由“白墙”与“黑洞”构成的体系。社会经济结构以及相应的权力组织形式的变化促成了“脸”的形成。 本文主要用“脸面性”思想来解读《分歧者》中的主人公翠丝，从她的“脸”经历出发，来探究主体如何通过“块茎”、“去疆域 化”、和“无器官身体”，最终线化“白墙”，冲出“黑洞”，实现个性的解放。

关键词: 《分歧者》；翠丝；“脸面性”；块茎；去疆域化无器官身体

\section{引言}

法国后现代主义哲学家吉尔・德勒兹是世界公认的隐喻大师, 在德勒兹与伽塔里的哲学体系中, “脸” 是其核心概念之一。人们为了共同利益或目的聚集在一起，自愿接受约定俗成的 “脸面性” 机器的 制约，无论人数多少，规模大小，都可以形成一个 “脸”集团。

“在德勒兹哲学体系中，“脸” 是一个由 “白墙”（white wall）与 “黑洞”（black hole）构成的 体系。“黑洞” 排列在白墙上, 洞口紧锁在白墙之上, 洞体横向无限延伸。“白墙” 是展示 “表征” （significance）的场所。“表征” 相当于 “能指” (signifier) 和 “所指” (signified) 之和。白墙 上所陈示的一切被称为 “圥謷” (redundancies)。“唯” 因其数量大、真正价值低，及其所起到的虚 饰作用而得其名。”（康有金＆朱碧荣，2015：65）

“脸” 本质上不是个性化的。脸面性系统在成员加入之前就已经剔除了不肯接受 “脸面性” 所认可的 核心思想的观点或成员（Deleuze＆Guattari 1987：168）。“脸” 体现出集体性，非个性化的社会团 体特点。“脸” 同时也具有排他性, 有共同目的或利益并能接受规约的脸员才可加入, 他者一律屏蔽。在 保护自身及所属集体利益上，“脸面性”（faciality）体现出人性化的一面，但对于他者，其非人性化 的一面则暴露无遗。“黑洞” 紧锁于 “白墙”之上，人的个性紧锁于 “黑洞”之中，压制人性主体。个性 
被迫变为虚无。“脸” 的本质属性圈起了利益范围, 同时也禁锢了人之个性。排他利已, 成为了一个非人 性化的压榨机器。

《分歧者》 (Divergent，2011)是美国青年小说家维罗妮卡 - 罗斯 (Veronica Roth，1988-) 的小说， 此部小说使她一举成名。《分歧者》这部小说的女主角翠丝, 生活在反乌托邦背景下的芝加哥, 那时的社 会被泾渭分明地分成了五个派别——友好派（Amity）、博学派（Erudite）、诚实派（Candor）、无私派 (Abnegation) 和无畏派 (Dauntless)。大家各司其职, 维护着世界和平, 而分歧者则不容于社会。小 说主人公翠丝兼具多种性格特质, 她是一名 “分歧者” , 其后, 小说叙述了她踏入反叛社会的路途, 并最 终推翻极权, 实现个性解放的历程。

对于这部作品的研究尚少。本文将借助德勒兹的 “脸面性” 理论, 结合文本, 来解读《分歧者》中的 主人公翠丝。

\section{1 翠丝之 “脸” 历程}

小说《分歧者》中的主人公翠丝经历的 “脸”历程主要有四个阶段，按照时间及过程的先后，它们分 别是 “在脸”、“去脸”、“入脸”及 “再去脸”。

每当经济结构发生变化, “脸面性机器” 就会发挥作用, 生产出一系列的 “脸”。对于小说中的未来 世界也不例外。在小说故事发生前, 世界的经济结构便已经发生了巨大变化。巨大的变化相应地带来了新 的权力组织及新的权力形式的产生。小说中的社会由此被划分为五个派别, 他们分别是: 崇尚友善, 抵制 侵略、冲突与战争的友好派; 崇尚知识, 抵制无知与愚昧的博学派; 崇尚正直, 抵制欺骗与谎言的诚实派; 崇尚奉献, 抵制冷漠与自私的无私派; 崇尚勇气, 抵制胆小与懦弱的无畏派。每派人都有自己所崇尚的、 突出的性格特征。社会中的每位成员都能在十六岁时根据自己的性格测试结果选择相应的派别, 并为自己 所选派别贡献余生。社会中所有人都被认定只拥有以上五种性格特质中的一种, 若兼具几种, 则被认定为 “分歧者”，“分歧者” 代表不被定义、不被操控，是破坏和平，制造动乱的代名词，为社会所不容。

兼具多种性格特质的翠丝则是社会所认定的潜在威胁者。表面上，整个社会是根据性格特质划分的， 实际上, 它是利益集团为了谋取各自利益, 掩盖各自实际目的的保护名。五个派别实质上就是五个 “脸” 集团, 他们吸附的是共同利益的寻求者, 为了获取最大利益, 这些寻求者自愿接受 “脸” 的规约与规制, 主动放弃个性, 向各自的 “脸” 集体靠拢。每个 “脸” 集团在利益互不冲突的情况下一团和气，维持着暗 藏汹涌波涛的表面和平。这样的社会是动荡的, 不堪一击的。翠丝作为五 “脸” 并存的社会一员, 她的存 在威胁到 “脸” 极权们的核心利益。所以, 她的社会生存不会是一帆风顺, 寻求个性解放的道路也将格外 艰辛。

勇敢、坚韧的翠丝不愿失去自由, 不愿失去主动性, 不愿随遇而安, 压迫个性, 至此, 她踏上了艰难 的去 “脸”历程。

\section{1 “在脸”}

生于无私派的翠丝，从小遵照着父母教导的准则生活。在无私派中，照镜子是虚荣的表现，准则规定 每一季度的第二天才可以照镜子, 此外, 所有的成员都整齐划一的穿着相同颜色的服装, 留着相同的发型, 每个成员好像都是同一人的无限复制。翠丝即是在这样的 “脸” 环境下成长。迷迷糊糊地接受着 “白墙” 之上的表征性，迷迷糊糊地接受着来自无私派 “脸” 组织的规制和约束，被动的将自己的主体性紧锁在 
“白墙”之上的 “黑洞”中。

此时, 翠丝还在成长, 自己的思想观念还在长芽, 父母的选择在这一阶段起着支配作用, 她无法选择 的、被动的 “在脸” 。

\section{2 “去脸” 与 “入脸”}

在小说《分歧者》中，年满十六周岁的社会成员将会参加能力测试，更确切的说是性格测试，测试完 成后, 根据测试结果和自己的考量, 被测者将在第二天的选派大会上决定自己想要加入并用余生为之奉献 的派别。绝大多数的被测者都会选择自己出生所在派别, 极少数会另择别派, 通常, 这些极少数会被原生 派别认为是反叛者。十六岁的翠丝在测试前, 已经有了自己的选择倾向, 她说; “我自私, 但我勇敢 ( I am selfish. I am brave. )”。测试结果表明自己是分歧者后, 她害怕并犹豫了。小说中, 哥哥迦勒曾多次 对翠丝说 “我们要考虑父母的感受, 但是, 我们更要听从自己的心声”。在选派大会上, 翠丝看到了哥哥 博学派的选择, 便下定决心, 选择了无畏派。内心的挣扎终于在此刻安定。这次选派的发生改变了翠丝的 命运，让她从束缚自己的 “黑洞” 中挣脱出来。选择了自己偏好的无畏派。

翠丝正在不断成长，比起 “在脸”时的買懂，现在的翠丝主体性开始被唤醒。但是，成长是需要时间 的, 翠丝 “去脸” 后, 进入了另一个 “脸” —无畏派。成长中的翠丝没有认清 “脸面化” 机器的本质。 根据自己的意志再次 “入脸” , 单纯的她认为, 紧锁自己的 “黑洞” 已完全打开, 封闭 “黑洞” 的白墙已 完全线化。实则不然, 无畏派是另一个被其核心成员操纵的非人性机器, 依旧压榨人的个性, 专制独裁, 一切齐整划一, 不容 “个性” 的个体。小说中, 托莉的哥哥在被无畏派 “脸” 核心发现是分歧者后无故消 失便是此最好的例证。

\section{3 “再去脸”}

翠丝入 “脸” 后, 经过对身体和心理上的魔鬼式训练, 她开始飞跃式的成长, 在发现博学派领导者珍 妮与无畏派领导者密谋要推翻无私派的领导时, 翠丝明白了自己的处境, 自己并没有逃脱出压抑自己的 “黑洞”，而是变成了 “脸” 利益集团的牺牲品，“白墙” 依旧紧紧的固着着 “黑洞”，迫使她完全接受 “白墙”上的 “资”，让她被同化为 “白墙”的一部分。

她开始意识到了 “白墙冗赘” 的存在, 被压制在 “黑洞” 中的主体性开始完全复活, 反抗的心情开始 急剧, 蓄积起拆除她所在 “脸” 的力量。最终, 通过自己的智慧、果敢和顽强, 挣脱了 “黑洞” , 线化了 “白墙”。实现了 “再去脸”。

\section{2 翠丝拆除 “脸” 的力量来源}

主体要从禁锢自己的 “白墙” 中解放, 从紧锁主体性的 “黑洞” 中挣脱, 需要超强的力量, 强大的力 量才能让个体线化 “白墙” , 冲出 “黑洞”。翠丝最终拆除 “脸” 的力量来自以下几个方面。

\section{1 “块茎” 的力量}

德勒兹和伽塔里从认识论角度出发, 创造了 “块茎” 这个概念。它是指从原母体上脱裂出来的两个子 事物, 其性质与母体的截然不同。块茎的生长遵循六条原则: 块茎的联系性、异质性、多样性、脱裂性、 反图绘性和反溯源性 (Deleuze \& Guattari 1987：7-12)。

翠丝的思维方式是 “块茎” 式, 即反常规的。她是德勒兹哲学思想中的 “少数派” 。“少数派” 的思 想是与 “多数派” 思想相对立的。“少数派” 不甘于被动接受现有的规约, 他们追求个性, 多样性, 追求 
真理而非恪守于统治者所假定的普遍性理念。这也是分歧者的原型。“多数派” 则与之相反, 他们遵照统 治者的意志和理念，接受现存的社会秩序。

翠丝敢于站出来质疑并挑战现有的制度规约, 用实际行动来摆脱非正义的束缚, 不畏人言与势力。这 是分歧者的特质, 也是 “块茎” 式思维方式的典型。

\section{2 “去疆域化” 的力量}

去疆域化即是主体通过逃逸, 离开旧有的环境进入新的环境, 在新环境中发掘自身的潜能, 营造出一 个全新自我的过程。去疆域化同时也是一个兼具发现与创造的过程。

小说主人公翠丝最终的个性解放便汲取了 “去疆域化” 的力量。她通过 “去脸”与 “再去脸” 两次逃 逸, 获取了力量源泉。在每次的去疆域化过程中, 她都再一次的认识了自己, 每去到一个新的环境, 都获 取了新的力量, 这是一个盘旋上升的前进过程。每次力量的获取, 都为她积聚起彻底冲出 “黑洞” 的力量。 在 “去脸” 来到无畏派后, 她重新认识了自己, 经过魔鬼式训练和情景测试, 她发现自己并不弱小, 而是 勇敢且坚㓞的, 母亲为她的改变也欣喜骄傲。训练场上, 翠丝在被彼得打倒后, 虽然全身负伤, 但是她并 没有放弃去参加野外实战训练, 而是忍着病痛, 追赶火车, 最后顺利完成训练并用智慧和毅力赢得了这场 比赛。

她超越了自己, 去疆域化让她在体力和智力上都获得了前所未有的增长, 为她最终冲出 “黑洞” 积攒 了巨大的力量。

\section{3 “无器官身体” 的力量}

在德勒兹的哲学体系中, “无器官身体” 也是其核心概念之一。根据德勒兹和伽塔里, “无器官身体” 是欲望的产物, 欲望在这里被定义为是支撑生活观念的 “生产性” 物质流, 是 “积极地”。他们把人看作 是欲望机器。每个人身上都存在 “无器官身体”, 其中, “无器官身体” 包含三种不同的组成成分, 它们 分别是恶化的 “无器官身体” 、干枯的 “无器官身体” 和完整的 “无器官身体” 。对于不同的人, 三种成 分所占比例不同。

在推翻极权统治和实现个性解放的驱动下, 翠丝的无器官身体总体上呈现的是完整的 “无器官身 体”。在宣扬 “派系高于亲情 (faction before blood) ” 的社会里, 亲人间可以因为派系利益而反目, 在这个世界里, 这是合情合理的。大家也都遵循着这假定好的社会规约, 但是, 翠丝并没有这样做, 她还 保留着同情心和人间亲情、友情。存留着完整的 “无器官身体”。她与四的爱情、父母间的亲情、与哥哥 的手足之情以及对于战友的感情都充分的证实了这一点。这也正是翠丝能获得最后成功的关键所在。

\section{3. 结语}

翠丝在 “块茎” 式思维的引导下, 经过 “去疆域化” , 成就了完整的 “无器官身体” , 各种力量的汇 聚, 让她顺利通过了 “去脸”、“入脸” 和 “再去脸” 的艰难过程, 完成了线化 “白墙” , 冲出 “黑洞”, 最后拆除 “脸” 的全过程。最终, 她成功地推翻了极权, 解放了个性, 让千疮百孔的世界重新闪炼起希望 的曙光。

\section{参考文献}

[1] Adrian P. The Deleuze Dictionary [M]. Scotland: Edinburgh University Press, 2005.

[2] Deleuze, G. and F. Guattari. Anti-Oedipus: Capitalism and Schizophrenia[M]. London: Continuum. 1983.

[3] Deleuze, G. and F. Guattari. A Thousand Plateaus: Capitalism and Schizophrenia[M]. London: University of Minnesota 
Press, 1987

[4] Roth, Veronica. Divergent[M]. New York: An Imprint of Harper Collins Publisher, 2012.

[5] Deleuze and Guattari. What Is Philosophy? [M]. Trans. Hugh Tomlinson.New York: Columbia University Press, 1994.

[6] Kang Youjin, Zhu Birong. 小说《白鲸》中埃哈伯人格的“脸面性”解读[J]. 当代外语研究, 2015(1): 65-69.

[7] [美] 维罗妮卡”罗斯. 分歧者[M]. 王明达译. 成都: 四川文艺出版社, 2014.

[8] 李尔萍, 康有金. 《拉帕西尼的女儿》的“脸面性”解读[J]. 长江大学学报(社科版), 2015(3): 35-37.

[9] 德勒兹: 《什么是生成?》[A]. 陈永国译. 汪民安主编. 生产(第五辑)[C]. 桂林: 广西师范大学出版社, 2008.

[10] 徐梦麟. 论《分歧者》中的选择与人性[J]. 青年文学家, 2015(17).

[11] 汪民安: 《文化研究关键词》 [M]. 南京: 江苏人民出版社, 2007.

\section{References:}

[1] Adrian P. The Deleuze Dictionary [M]. Scotland: Edinburgh University Press, 2005.

[2] Deleuze, G. and F. Guattari. Anti-Oedipus: Capitalism and Schizophrenia [M]. London: Continuum. 1983.

[3] Deleuze, G. and F. Guattari. A Thousand Plateaus: Capitalism and Schizophrenia [M]. London: University of Minnesota Press, 1987.

[4] Roth, Veronica. Divergent [M]. New York: An Imprint of Harper Collins Publisher, 2012.

[5] Deleuze and Guattari. What Is Philosophy? [M]. Trans. Hugh Tomlinson. New York: Columbia University Press, 1994.

[6] Kang Youjin,Zhu Birong. Personality "Face Nature" Interpretation of Ahab in Novel White Whale [J].Contemporary Foreign Language studies, 2015(1):65-69.

[7] [US] Veronica Ross. Divergent [M].Wang Mingda translation. Chengdu: Sichuan Literature and Art Publishing House, 2014.

[8] Li Erping, Kang Youjin. "Face Nature" Interpretation of Rappaccini's Daughter [J].Journal of Yangtze University (Social Sciences Edition), 2015(3):35-37.

[9] Deleuze: What is generated? [A].Chen Yongguo translation. Wang Minan editor. Production (The Fifth Series) [C].Guilin: Guangxi Normal University Press, 2008.

[10] Xu Menglin. On selection and Human Nature in Divergent [J]. Youth Literator, 2015(17).

[11] Wang Min'an Cultural Research Keywords [M].Nanjing: Jiangsu People's Publishing Ltd, 2007. 